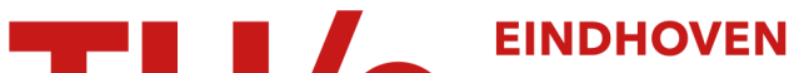 UNIVERSITY OF TECHNOLOGY
}

\section{Loss compensation of extraordinary optical transmission}

\section{Citation for published version (APA):}

Beijnum, van, F., Veldhoven, van, P. J., Geluk, E. J., Hooft, 't, G. W., \& Exter, van, M. P. (2014). Loss compensation of extraordinary optical transmission. Applied Physics Letters, 104, 061112-1/3. [061112]. https://doi.org/10.1063/1.4865416

DOI:

$10.1063 / 1.4865416$

Document status and date:

Published: 01/01/2014

\section{Document Version:}

Publisher's PDF, also known as Version of Record (includes final page, issue and volume numbers)

\section{Please check the document version of this publication:}

- A submitted manuscript is the version of the article upon submission and before peer-review. There can be important differences between the submitted version and the official published version of record. People interested in the research are advised to contact the author for the final version of the publication, or visit the $\mathrm{DOI}$ to the publisher's website.

- The final author version and the galley proof are versions of the publication after peer review.

- The final published version features the final layout of the paper including the volume, issue and page numbers.

Link to publication

\section{General rights}

Copyright and moral rights for the publications made accessible in the public portal are retained by the authors and/or other copyright owners and it is a condition of accessing publications that users recognise and abide by the legal requirements associated with these rights.

- Users may download and print one copy of any publication from the public portal for the purpose of private study or research.

- You may not further distribute the material or use it for any profit-making activity or commercial gain

- You may freely distribute the URL identifying the publication in the public portal.

If the publication is distributed under the terms of Article 25fa of the Dutch Copyright Act, indicated by the "Taverne" license above, please follow below link for the End User Agreement:

www.tue.nl/taverne

Take down policy

If you believe that this document breaches copyright please contact us at:

openaccess@tue.nl

providing details and we will investigate your claim. 


\title{
Loss compensation of extraordinary optical transmission
}

\author{
Frerik van Beijnum, ${ }^{1}$ Peter J. van Veldhoven, ${ }^{2}$ Erik Jan Geluk, ${ }^{2}$ Gert W. 't Hooft, ${ }^{1,3}$ \\ and Martin P. van Exter ${ }^{1}$ \\ ${ }^{1}$ Leiden University, Huygens Laboratory, P.O. Box 9504, 2300 RA Leiden, The Netherlands \\ ${ }^{2}$ COBRA Research Institute, Technische Universiteit Eindhoven, Postbus 513, 5600 MB Eindhoven, \\ The Netherlands \\ ${ }^{3}$ Philips Research Laboratories, Prof. Holstlaan 4, 5656 AA Eindhoven, Netherlands
}

(Received 26 November 2013; accepted 19 January 2014; published online 12 February 2014)

\begin{abstract}
In the past decade, metal hole arrays have been studied intensively in the context of extraordinary optical transmission (EOT). Recently it was shown that surface plasmons on optically pumped hole arrays can show laser action. So far, however, it is not demonstrated that the optical transmission of these arrays can also be increased using gain. In this Letter, we present a dramatic increase of the EOT via loss compensation of surface plasmons, accompanied by spectral narrowing of the resonance. These experiments allow us to quantify the modal gain experienced by the surface plasmon. Interestingly, the transmission minimum of the Fano-resonance becomes smaller. (C) 2014 AIP Publishing LLC. [http://dx.doi.org/10.1063/1.4865416]
\end{abstract}

Surface plasmons offer many possibilities in photonics, but applications are often limited by absorption or radiative loss. Hence, compensating these losses will further improve the applicability of surface plasmons. In this context, long range surface plasmons have been amplified, ${ }^{1,2}$ and amplified spontaneous emission of surface plasmons is reported. ${ }^{3,4}$ Simultaneously, various kinds of surface plasmon lasers ${ }^{5}$ have been studied experimentally. ${ }^{6-11}$ Despite the large interest in surface plasmon lasers, lasing may also be a nuisance when improving a lossy metallic system. Such problems may occur in metamaterials, ${ }^{12}$ like negative index materials ${ }^{13-15}$ or $\mathrm{n}=0$ metamaterials. ${ }^{16}$

A metal hole array, which is known for its extraordinary optical transmission (EOT), ${ }^{17}$ is an ideal system to study loss compensation and lasing for various reasons. EOT is thoroughly studied ${ }^{18}$ and the role of surface plasmons is also understood quantitatively. ${ }^{19,20}$ EOT also has potential applications, like biosensing. ${ }^{21,22}$ Finally, EOT is an example of a Fano resonance, ${ }^{23}$ often exploited in plasmonic nanostructures. ${ }^{24-27}$

Surface plasmon lasing was recently reported in metal hole arrays,${ }^{10}$ thereby showing that surface plasmons in this structure can exist without loss. The hole array lases in a subradiant mode, which is a resonance with limited radiative loss. In this Letter we study the effect loss compensation by optical gain on the EOT. Because the optical transmission is carried by radiant modes, the losses are expected to be much larger ${ }^{11,28-30}$ than that of the lasing mode. The experiments reported in this Letter show loss compensation of the surface plasmons, through a strong enhancement of the peak transmission and a spectral narrowing of the resonance, and quantify these effects.

Figure 1 shows the essence of the experiment, which is measuring the optical transmission of a metal hole array on a semiconductor (InGaAs) gain layer. The gain layer is optically pumped with a $1064 \mathrm{~nm}$ laser beam. The experiment is performed in a Helium flow cryostat to increase the potential gain of the semiconductor. The (extraordinary) optical transmission spectrum is measured with a white light source that is spectrally analyzed after transmission through the hole array. We study the influence of gain on the (surface plasmon resonance in the) EOT spectrum. The luminescence of the gain material, which is also transmitted through the holes and emitted in all directions (red arrows), gives rise to background luminescence.

Some technical details: the sample is illuminated with two beams, the pump beam is $\sim 40 \mu \mathrm{m}$ diameter, and the white light signal $\sim 12 \mu \mathrm{m}$ diameter. To create a pump spot of uniform intensity we illuminated a pinhole with the pump laser, to select the central $25 \%$ power, and imaged this pinhole on the sample. The signal beam is generated using a super-luminescent diode with center wavelength $1550 \mathrm{~nm}$ and spectral width of $110 \mathrm{~nm}$. The transmitted light is collected with a microscope objective. The far field of the objective is imaged onto a single mode fiber, which is subsequently led to a grating spectrometer with a linear array. The angular resolution of this setup is $\sim 4 \mathrm{mrad}$. The collected laser emission from the array is minimized by positioning of the detecting fiber close to an intensity minimum of the donut-shaped laser emission.

The absolute transmission is determined as follows. Because the sample is placed inside a cryostat, we could not

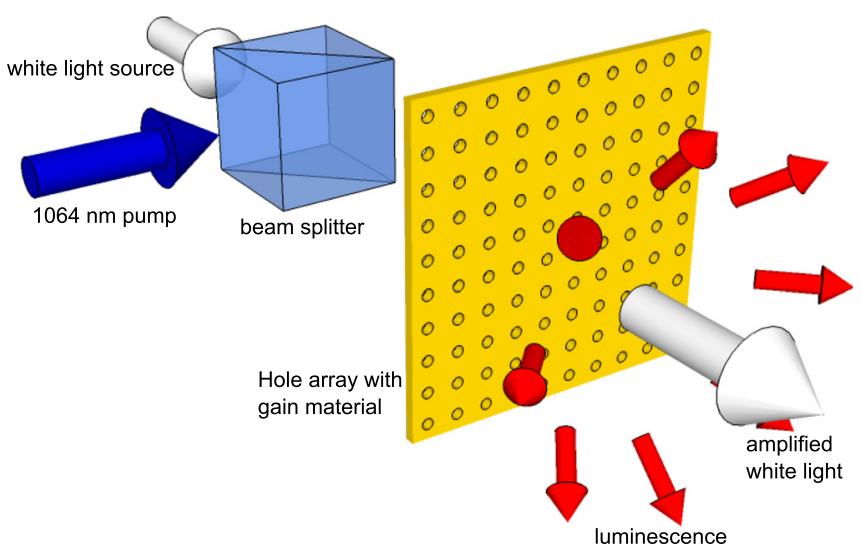

FIG. 1. Sketch of the experiment. We record the white light transmission spectrum of a hole array that has a gain layer in close proximity of the metal, while pumping the gain layer with a laser. The luminescence from the pumped gain material is also recorded (red arrows). 
move the sample in and out of the beam without changing the alignment. For this reason, we first used the signal at unperforated areas of the sample as a reference. Later, we measured transmission spectra of these areas at room temperature and without optical pumping, both inside and outside the cryostat. As the later measurement was easy to quantify, we thus found the absolution transmission of the sample inside the cryostat.

The sample is fabricated as follows. On a semiinsulating indium phosphide wafer a lattice-matched indium gallium arsenide layer is grown $(105 \mathrm{~nm})$, which is subsequently covered with a thin $(15 \mathrm{~nm})$ layer of InP. Hereafter a $5 \mathrm{~nm}$ protective silicon nitride layer is grown using plasma enhanced chemical vapor deposition. On these layers, we fabricate the metal hole array by depositing $100 \mathrm{~nm}$ gold and $20 \mathrm{~nm}$ chrome on a lithographically defined array of dielectric pillars. To provide sufficient adhesion of the gold onto the Silicon Nitride, we deposit a very thin (average thickness smaller than $0.5 \mathrm{~nm}$ ) chromium adhesion layer in between these layers. The last step is to etch the pillars away, leaving the subwavelength holes (diameters ranging from $180 \mathrm{~nm}$ to $190 \mathrm{~nm})$.

Figure 2 shows the key result of this Letter, namely the evolution of the transmission spectrum with pump power. The inset shows the transmission when the system is not pumped (red curve). This spectrum shows the well-known Fano line shape, with a transmission maximum of $8.6 \times 10^{-3}$. The dashed grey curve shows the estimated transmission in the absence of any surface waves, which is $2.3 \times 10^{-3}$ at $1500 \mathrm{~nm}$. Hence the transmission of the unpumped system is enhanced by a factor 3.7 by the presence of surface waves.

When we increase the pump power the maximum transmission increases dramatically, while it remains practically constant at other wavelengths. At $90 \mathrm{~mW}$ pump power, the transmission has reached a value of 0.25 , corresponding to a 31 fold increase of the transmission. The transmission enhancement as a result of surface waves is now 109, instead of 3.7. As a reference, in a recent experiment without loss compensation we found an 17 fold enhancement for a hole array on glass. ${ }^{20}$ Hence we conclude that loss compensation

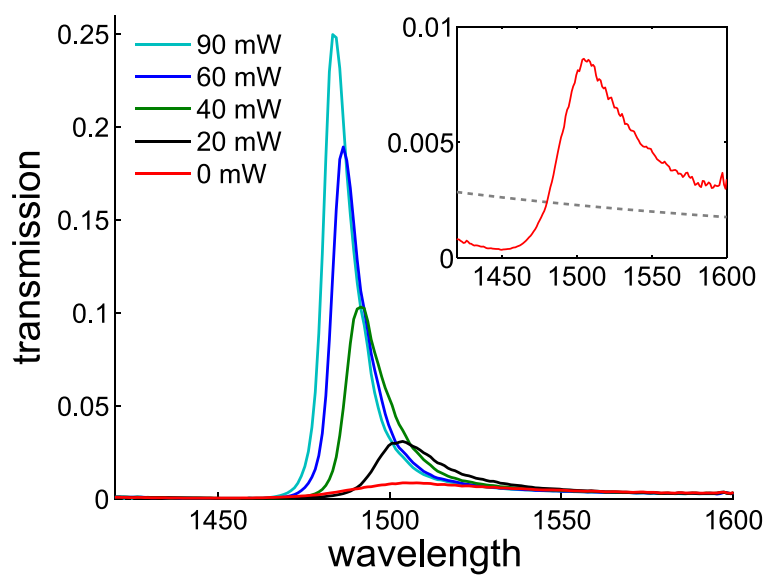

FIG. 2. Measured transmission as a function of pump power. The transmission maximum gradually increases with pump power from $8.6 \times 10^{-3}$ (see inset) to 0.25 , which is a 31 fold enhancement. The lattice spacing of this sample is $a_{0}=450 \mathrm{~nm} ; 90 \mathrm{~mW}$ corresponds to roughly $7 \mathrm{~kW} / \mathrm{cm}^{2}$ can dramatically increase the EOT. Enhanced transmission and spectral narrowing of the transmission resonance is a generic feature that has a.o. also been predicted for optical metamaterials built from metal and a dielectric gain medium. ${ }^{31}$

In Figure 3, we compare the amplified white-light transmission with the background luminescence. The maximum transmission is much $(\sim 10 \times)$ larger than the background luminescence at resonance. The transmission plotted here is $T=\left(I_{\text {out }}-I_{b g}\right) / I_{i n}$, where $I_{\text {in }}$ is the incident white light intensity, $I_{b g}$ is the background signal when only the pump is switched on, and $I_{\text {out }}$ is the intensity recorded when both light sources are on. The background luminescence plotted in Fig. 3 is $I_{b g}$ divided by the value of $I_{i n}$ at $1490 \mathrm{~nm}$. The background luminescence is not affected by the white light source, which we checked by also studying the background luminescence at an angle where the white light is absent.

At the transmission minimum the background signal exceeds the transmitted signal. This imposes a challenge on measuring the transmission minimum accurately. To limit this problem, the light source used in a loss compensated transmission experiment has to be sufficiently bright. Hence we used a superluminescent diode for these experiments, instead of the halogen lamp that is often used for EOT experiments.

We will first discuss the background luminescence, before discussing the more important transmission spectrum. The background luminescence is well fitted by a Lorentzian lineshape (dashed curve). The resonance wavelength and spectral width of this Lorentzian is the same as that of the Fano resonance that is fitted to the transmission spectrum (solid curve). This shows that both resonances have the same origin, namely surface waves propagating on the metal dielectric interface.

The background luminescence in Figure 3 exhibits two narrow resonances at $\sim 1425 \mathrm{~nm}$ and $\sim 1460 \mathrm{~nm}$. These are two other resonances with a subradiant character. ${ }^{29}$ The transmission experiments reported here are performed slightly above the lasing threshold of these modes. ${ }^{10}$ The recorded laser intensity remains modest, because the

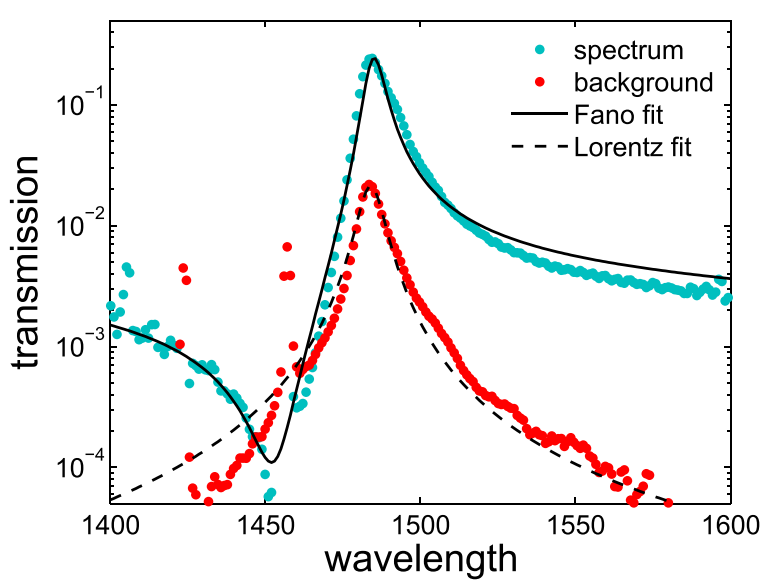

FIG. 3. Comparison between the collected background luminescence and the transmitted white light. At the transmission maximum, the background luminescence is an order of magnitude smaller. At the transmission minimum, however, the magnitude of the background luminescence exceeds that of the transmitted white light. Please note that the plots are on a semilog scale. 

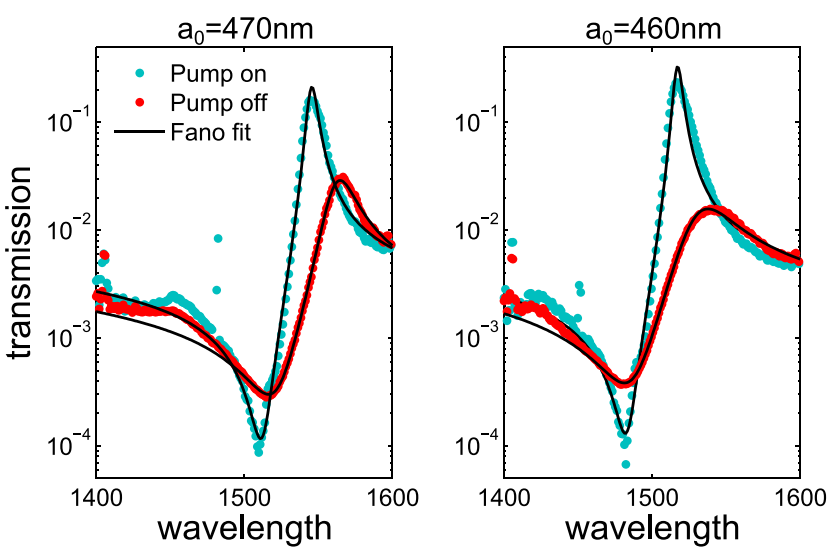

transmission experiment is performed around the surface normal, in the intensity minimum of the donut-shaped laser emission. ${ }^{10}$ Nonetheless, the laser action may hinder loss compensation in two ways. First, the lasing peak will largely exceed the transmitted signal, when the device is operated far above threshold, and background subtraction becomes inaccurate. Second, when the device starts lasing the gain of the semiconductor will not increase anymore with a further increase of pump power, because all additional pump power is lost to the lasing mode. ${ }^{4,32}$ Hence, these two narrow resonances exemplify that loss compensation can lead to lasing effects at one wavelength that hinder further loss compensation at other wavelengths. ${ }^{12}$

In Fig. 4 we compare transmission spectra of three samples with different lattice spacings $a_{0}=470,460$, and $450 \mathrm{~nm}$, of which the $450 \mathrm{~nm}$ sample was discussed in Figures 2 and 3. The transmission spectra of these three samples, with and without pumping, are plotted on a semilog scale. Each sample is pumped strong enough to be close to full inversion within the bandwidth of the Fano resonances. Noteworthy is that the transmission at the minimum decreases in the presence of the pump, showing that pumping can also decrease the transmission! This experimental observation is consistent with simulations of the EOT (rigorous coupled wave analysis) for different surface plasmon losses. The observed decrease indicates that the destructive interference between the resonant and non-resonant transmission becomes more complete in the presence of gain. The quantitative analysis presented below will address two questions: "How much gain is supplied by the semiconductor material?" and "What limits the observed enhancement?"

The six smooth curves in Fig. 4 are Fano fits to the data. Our Fano expression is an approximate version of a microscopic model for EOT, which was recently developed ${ }^{19}$ and verified experimentally. ${ }^{20}$ The expression for these fits is

$$
T(\omega)=\left|t \omega^{2}+\frac{\alpha \omega^{4}}{\omega-\omega_{0}+i \gamma}\right|^{2},
$$

where $t$ is proportional to the transmission in absence of surface waves, $\alpha$ quantifies the combined excitation and outcoupling $\left(\alpha / t\right.$ can be complex), $\omega_{0}$ is the resonance frequency, and $\gamma$ is the combined ohmic and radiative losses of the surface plasmon. For convenience we expressed $\omega, \omega_{0}$ and $\gamma$ in $\mathrm{eV}$.

In Table I we show the fit values used for the six fits in Figure 4. The second column shows that the resonance

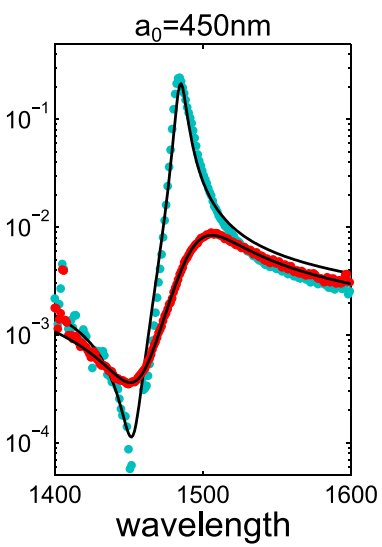

FIG. 4. Transmission for samples of different lattice spacing. From left to right the spacing is $a_{0}=470 \mathrm{~nm}$, $460 \mathrm{~nm}$, and $450 \mathrm{~nm}$. The transmission at maximum pump power $(90 \mathrm{~mW})$ is compared to that without pumping. The data is fitted to a Fano resonance.

wavelength $\lambda_{0}$ is proportional to $a_{0}$ and decreases by $10-15 \mathrm{~nm}$ under the influence of optical pumping, as expected. The third column shows the linewidth $\Delta \lambda$. This is the most important parameter as it quantifies the surface plasmon loss. For the unpumped samples, the linewidth increases from $\Delta \lambda=12 \mathrm{~nm}$ at $\lambda_{0}=1562 \mathrm{~nm}$ to $\Delta \lambda=24 \mathrm{~nm}$ at $\lambda_{0}=1494 \mathrm{~nm}$. This is as expected: at smaller wavelengths the absorption losses in the gold and the unpumped gain medium increase, while the scattering losses induced by the holes also increase. For the pumped samples, all linewidths are $\Delta \lambda \sim 4 \mathrm{~nm}$. The reason for this similarity is not yet clear.

The difference between the pumped and unpumped linewidth, which increases from 8 to $20 \mathrm{~nm}$ when $a_{0}$ decreases, is proportional to the gain experienced by the surface plasmon. The associated change in inverse propagation length of the surface plasmon is

$$
\frac{4 \pi n_{\mathrm{eff}}}{\lambda^{2}}\left(\Delta \lambda_{\text {off }}-\Delta \lambda_{\text {on }}\right)=L_{\text {off }}^{-1}-L_{\text {on }}^{-1},
$$

where $L_{\text {off }}$ and $L_{\text {on }}$ are the inverse propagation lengths without and with pump, respectively. The difference $L_{\text {off }}^{-1}-L_{\text {on }}^{-1}$ is $3.5 \times 10^{3} \mathrm{~cm}^{-1}$ for the $a_{0}=450 \mathrm{~nm}$ sample. To convert this change into a value for the material gain of the semiconductor, we (i) divide by a factor two, to account for the transition from semiconductor loss to gain and (ii) divide by the optical confinement factor of the surface plasmon mode, which we estimated to be 0.32 for our system. Hence, the estimated material gain is $5.5 \times 10^{3} \mathrm{~cm}^{-1}$ for the $450 \mathrm{~nm}$ sample and $2.2 \times 10^{3} \mathrm{~cm}^{-1}$ for the $470 \mathrm{~nm}$ sample. These numbers are reasonable for a semiconductor operated at high carrier densities and low temperatures. ${ }^{8,33}$ Even with this high gain, the direct transmission through the $105 \mathrm{~nm}$ semiconductor layer increases only by $6 \%$ from unpumped to

TABLE I. Fit parameters corresponding to the Fano fits in Figure 4. Dimensions of $\lambda_{0}$ and $\Delta \lambda$ are nm. Dimensions of $|\alpha|$ and $t$ are $\mathrm{eV}^{-4}$ and $\mathrm{eV}^{-2}$, respectively.

\begin{tabular}{llllc}
\hline \hline Parameter & $\lambda_{0}$ & $\Delta \lambda$ & $|\alpha|$ & $t$ \\
\hline $470 \mathrm{~nm}$, off & 1562 & 12 & $2.8 \times 10^{-3}$ & $73 \times 10^{-3}$ \\
$470 \mathrm{~nm}$, on & 1545 & 4 & $2.4 \times 10^{-3}$ & $87 \times 10^{-3}$ \\
$460 \mathrm{~nm}$, off & 1530 & 20 & $2.7 \times 10^{-3}$ & $80 \times 10^{-3}$ \\
$460 \mathrm{~nm}$, on & 1517 & 4 & $2.5 \times 10^{-3}$ & $90 \times 10^{-3}$ \\
$450 \mathrm{~nm}$, off & 1494 & 24 & $2.1 \times 10^{-3}$ & $70 \times 10^{-3}$ \\
$450 \mathrm{~nm}$, on & 1485 & 4 & $2.1 \times 10^{-3}$ & $81 \times 10^{-3}$ \\
\hline \hline
\end{tabular}


pumped; an effect that can easily be neglected and hardly shows up in (the value of $t$ in) Table I.

The parameter $\alpha$, which models the excitation and outcoupling of the surface plasmons, hardly depends on pump power for each sample. This shows that the measured transmission enhancement and spectral narrowing are consistent with the expected $T_{\max } \approx|\alpha|^{2} \omega^{8} / \gamma^{2}$. The parameter $t$ is also more or less constant for all six cases, as expected for the non-resonant transmission.

Finally, we note that there is room for improvement. The surface plasmon gain can for instance be increased by carefully maximizing the thickness of the gain layer or placing the gain layer closer to the metal interface. The system would also become more practical when electrical pumping is implemented and when the structures can be operated at room temperature.

In conclusion, we have demonstrated the improved performance of a plasmonic system using a semiconductor gain material. We increase the transmission of a metal hole array by a factor 31 , while the transmission minimum decreases. Our quantitative analysis shows that we experimentally obtain the expected large material gain. Two challenges are identified that are generic to loss compensation in plasmonic systems with Fano resonances: (1) subtraction of the background luminescence can be troublesome, in particular when the transmission minimum decreases under loss compensation. To observe any transmission signal, a very intense signal beam is required compared to systems without gain; (2) gain saturation will occur when the structure lases unintentionally. Therefore, loss compensated systems need to be designed with much care, taking the physics of the gain medium and other optical resonances into account.

We acknowledge M. T. Hill, M. J. H. Marell, E. Smalbrugge, and T. de Vries for discussions. This work is part of the research program of the Foundation for Fundamental Research on Matter (FOM), which is part of the Netherlands Organisation for Scientific Research (NWO).

${ }^{1}$ M. C. Gather, K. Meerholz, N. Danz, and K. Leosson, Nat. Photon. 4, 457 (2010).

${ }^{2}$ I. D. Leon and P. Berini, Nat. Photon. 4, 382 (2010).

${ }^{3}$ M. Noginov, G. Zhu, M. Mayy, B. Ritzo, N. Noginova, and V. Podolskiy, Phys. Rev. Lett. 101, 226806 (2008).
${ }^{4}$ P. M. Bolger, W. Dickson, A. V. Krasavin, L. Liebscher, S. G. Hickey, D. V. Skryabin, and A. V. Zayats, Opt. Lett. 35, 1197 (2010).

${ }^{5}$ D. J. Bergman and M. I. Stockman, Phys. Rev. Lett. 90, 027402 (2003).

${ }^{6}$ M. A. Noginov, G. Zhu, A. M. Belgrave, R. Bakker, V. M. Shalaev, E. E. Narimanov, S. Stout, E. Herz, T. Suteewong, and U. Wiesner, Nature 460, 1110 (2009)

${ }^{7}$ R. F. Oulton, V. J. Sorger, T. Zentgraf, R.-M. Ma, C. Gladden, L. Dai, G. Bartal, and X. Zhang, Nature 461, 629 (2009).

${ }^{8}$ P. Berini and I. De Leon, Nat. Photon. 6, 16 (2011).

${ }^{9}$ R. M. Ma, R. F. Oulton, V. J. Sorger, and X. Zhang, Laser Photonics Rev. 7, 1 (2013).

${ }^{10}$ F. van Beijnum, P. J. van Veldhoven, E. J. Geluk, M. J. A. de Dood, G. W. 't Hooft, and M. P. van Exter, Phys. Rev. Lett. 110, 206802 (2013).

${ }^{11}$ M. P. van Exter, V. T. Tenner, F. van Beijnum, M. J. A. de Dood, P. J. van Veldhoven, E. J. Geluk, and G. W. 't Hooft, Opt. Express 21, 27422 (2013).

${ }^{12}$ M. I. Stockman, Phys. Rev. Lett. 106, 156802 (2011).

${ }^{13}$ S. Zhang, W. Fan, N. C. Panoiu, K. J. Malloy, R. M. Osgood, and S. R. J. Brueck, Phys. Rev. Lett. 95, 137404 (2005).

${ }^{14}$ G. Dolling, M. Wegener, C. M. Soukoulis, and S. Linden, Opt. Lett. 32, 53 (2007).

${ }^{15}$ S. Xiao, V. P. Drachev, A. V. Kildishev, X. Ni, U. K. Chettiar, H.-K. Yuan, and V. M. Shalaev, Nature 466, 735 (2010).

${ }^{16}$ E. Jan, T. Coenen, H. Caglayan, N. Engheta, and A. Polman, Phys. Rev. Lett. 110, 013902 (2013).

${ }^{17}$ T. W. Ebbesen, H. J. Lezec, H. F. Ghaemi, T. Thio, and P. A. Wolff, Nature 391, 667 (1998).

${ }^{18}$ F. J. García-Vidal, L. Martín-Moreno, T. W. Ebbesen, and L. Kuipers, Rev. Mod. Phys. 82, 729 (2010).

${ }^{19}$ H. T. Liu and P. Lalanne, Nature 452, 728 (2008).

${ }^{20}$ F. van Beijnum, C. Retif, C. B. Smiet, H. Liu, P. Lalanne, and M. P. van Exter, Nature 492, 411 (2012).

${ }^{21}$ A. G. Brolo, R. Gordon, B. Leathem, and K. L. Kavanagh, Langmuir 20, 4813 (2004).

${ }^{22}$ A. A. Yanik, M. Huang, O. Kamohara, A. Artar, T. W. Geisbert, J. H. Connor, and H. Altug, Nano Lett. 10, 4962 (2010).

${ }^{23}$ U. Fano, Phys. Rev. 124, 1866 (1961).

${ }^{24}$ C. Genet, M. P. van Exter, and J. P. Woerdman, Opt. Commun. 225, 331 (2003).

${ }^{25}$ A. E. Miroshnichenko, S. Flach, and Y. S. Kivshar, Revi. Mod. Phys. 82, 2257 (2010).

${ }^{26}$ B. Luk'yanchuk, N. I. Zheludev, S. A. Maier, N. J. Halas, P. Nordlander, H. Giessen, and C. T. Chong, Nat Mater. 9, 707 (2010).

${ }^{27}$ M. Rahmani, B. Luk'yanchuk, and M. Hong, Laser Photonics Rev. 7, 329 (2013).

${ }^{28}$ P. Paddon and J. F. Young, Phys. Rev. B 61, 2090 (2000).

${ }^{29}$ C. Ropers, D. J. Park, G. Stibenz, G. Steinmeyer, J. Kim, D. S. Kim, and C. Lienau, Phys. Rev. Lett. 94, 113901 (2005).

${ }^{30}$ M. J. A. de Dood, E. F. C. Driessen, D. Stolwijk, and M. P. van Exter, Phys. Rev. B 77, 115437 (2008).

${ }^{31}$ J. Yang, C. Sauvan, H. T. Liu, and P. Lalanne, Phys. Rev. Lett. 107, 043903 (2011).

${ }^{32}$ R. E. Slusher, A. F. J. Levi, U. Mohideen, S. L. McCall, S. J. Pearton, and R. A. Logan, Appl. Phys. Lett. 63, 1310 (1993).

${ }^{33}$ M. T. Hill, J. Opt. Soc. Am. B 27, B36 (2010). 